Original Research Paper

\title{
Determination of Neuromuscular Diseases Using Complexity of Electromyogram Signals
}

\author{
${ }^{1}$ Kawser Ahammed and ${ }^{2}$ Mosabber Uddin Ahmed \\ ${ }^{1}$ Department of Electrical and Electronic Engineering, \\ Jatiya Kabi Kazi Nazrul Islam University, Trishal, Mymensingh, Bangladesh \\ ${ }^{2}$ Department of Electrical and Electronic Engineering, University of Dhaka, Dhaka, Bangladesh
}

\author{
Article history \\ Received: 10-10-2019 \\ Revised: 29-11-2019 \\ Accepted: 06-12-2019 \\ Corresponding Author: \\ Kawser Ahammed \\ Department of Electrical and \\ Electronic Engineering, \\ Jatiya Kabi Kazi Nazrul \\ Islam University, Trishal, \\ Mymensingh, Bangladesh \\ Email: kawser@jkkniu.edu.bd
}

\begin{abstract}
Characterization of Electromyogram (EMG) signals is important for identifying neuromuscular diseases. Although various techniques were implemented for classification, none of them were implemented in the complexity domain. In this study, characterization of EMG signals recorded from healthy, neuropathic and myopathic subjects has been performed in complexity domain based on Multiscale Entropy (MSE) method. To do this, the multiscale entropy method has been applied to an EMG database publicly available in PhysioNet. The complexity profile curves obtained with the MSE approach have shown promising classification among healthy subject, neuropathic and myopathic patients in terms of complexity. One way ANOVA test has shown statistically significant differences $(p<0.01)$ among these three classes. Moreover, Support Vector Machine (SVM) has demonstrated a classification accuracy of $86.1 \%$ for characterizing EMG signals of neuromuscular disorders. Furthermore, myopathic and neuropathic patients have been recognized with $66.7 \%$ sensitivity at $95.8 \%$ specificity and $100 \%$ sensitivity at $100 \%$ specificity respectively.
\end{abstract}

Keywords: Electromyogram, Neuromuscular Diseases, Complexity, Multiscale Entropy, Support Vector Machine

\section{Introduction}

Neuromuscular diseases are disorders that affect nerve or muscle, neuromuscular junctions, and muscle tissue. Neuropathy and myopathy are two types of neuromuscular diseases. When motoneuron cells involved in muscular control or sensation (Brown et al., 2002; Basmajian and De Luca, 1985; Kandel et al., 2000) die, patients suffer from neuropathy. Patients suffer from myopathy when the muscle fibers die (Kocer, 2010). Therefore, automatic and correct characterization of these diseases is necessary for a clinical environment.

Although the electromyography test is used for the diagnosis of neuromuscular disorders, this technique is subjective (Subasi, 2013). Because the signals acquired in this type of test are analyzed using a visual and audible way by an adroit electrophysiologist (Kimura, 2013), this subjectivity test should be replaced with automatic classification techniques to help the diagnosis (Subasi, 2013).

Various classification techniques including multilayer perceptrons (Christodoulou and Pattichis, 1999), neural networks (Abel et al., 1996; Pandey and Mishra, 2009; Vallejo et al., 2018), fuzzy approaches (Hamilton-Wright et al., 2007), support vector machines (Katsis et al., 2006), neuro-fuzzy systems (Kocer, 2010) and fuzzy entropies (Vallejo et al., 2018) were employed by several studies for detecting neuromuscular diseases. However, although all of the approaches have shown promising classification accuracy, none of them were implemented in the complexity domain for classifying EMG signals in terms of complexity. Although Istenič et al. (2010) proposed a multiscale entropy-based feature extraction approach for classifying neuromuscular disorders, this approach characterized the neuromuscular disorders 
depending on the amplitude distribution of the surface EMG rather than complexity. Moreover, the provided classification accuracy $(70.4 \%)$ for three classes (healthy, neuropathic and myopathic) was not promising.

In this study, a complexity analysis method based on the MSE approach has been used for classifying signals collected from patients suffering from neuropathy, signals recorded from myopathic patient and signals extracted from a healthy subject. The goal of this classification is to detect neuromuscular diseases in a clinical environment with multiscale entropy of EMG signals.

\section{Materials and Methods}

\section{Database}

The Database used in this research is publicly available at PhysioNet (Goldberger et al., 2003). The database contains EMG samples recorded from tibialis anterior muscle of each healthy, neuropathy and myopathy patients using a 25-millimeter concentric needle electrode and a Synergy N2 EMG Monitoring System. The healthy database consisted of a fortyfour-year-old subject showing no symptoms of neuromuscular diseases. The neuropathy set was made up of a sixty-two-year-old subject representing longlasting low back pain and symptoms of neuropathy. The remaining set comprised of a fifty-seven-year-old subject demonstrating myopathy. During the recording process, the frequency of data was $50 \mathrm{kHz}$ and then downsampled to $4 \mathrm{kHz}$. While recording a high pass filter and a low pass filter designed at $20 \mathrm{~Hz}$ and $5 \mathrm{kHz}$ respectively were also used. Fig. 1 shows EMG signals from this dataset. In this work, we have used 12 subsets of EMG data from each class. Each subset contains 4000 samples.

\section{Multiscale Entropy}

The Multiscale Entropy approach (MSE) (Costa et al., 2002) determines Sample Entropy (SamEn) (Richman and Moorman, 2000) over different time scales to characterize the underlying complexity of nonlinear time series. For calculating the SamEn, the MSE method includes three parameters- $\tau$ (time lags), $m$ (embedding dimensions) and $r$ (threshold value). The MSE analysis consists of the following two steps:

- To define temporal scales of increasing length, a coarse-graining process to the following time series was applied:

$$
\left\{u_{i}\right\}_{i=1}^{N}
$$

where $N$ denotes the number of samples in the time series. For a scale factor $\xi$, the resulting coarsegrained time series is calculated as:

$$
x_{j}^{\xi}=\frac{1}{\xi} \sum_{i=(j-1) \xi+1}^{j \xi} u_{i}
$$

where $1 \leq j \leq \frac{N}{\xi}$.

- To plot sample entropy as a function of scale factor $\xi$, follow algorithm 1 and calculate sample entropy for each coarse-grained time series $x_{j}^{\xi}$.

\section{Algorithm 1: Sample Entropy}

- Form $(N-m)$ vectors $U_{m}(1), U_{m}(2), \ldots, U_{m}(N-m)$ defined by $U_{m}(i)=\left[u_{m}(i), u_{m}(i+1), \ldots, u_{m}(i+m-1)\right]$, where $i=1,2, \ldots, N-m$.

- $\quad$ Determine the distance between two vectors $U_{m}(i)$ and $U_{m}(j)$ as maximum norm $d\left[U_{m}(i), U_{m}(j)\right]=\max _{k}$ $=1, \ldots, m\{|u(i+k-1)-u(j+k-1)|\}$.

- Estimate the frequency of occurrence as $A_{i}^{m}(r)=\frac{1}{N-m-1} A_{i}$ and define a global quantity, $A^{m}(r)=\frac{1}{N-m} \sum_{i=1}^{N-m} A_{i}^{m}(r)$, where $d\left[U_{m}(i), U_{m}(j)\right] \leq$ $r, j \neq i, r$ denotes a threshold value.

- Extend the dimension of the vectors to $m+1$ and calculate the frequency of occurrence $A_{i}^{m+1}(r)=\frac{1}{N-m-1} A_{i}$ and define a global quantity $A^{m+1}(r)=\frac{1}{(N-m)} \sum_{i=1}^{(N-m)} A_{i}^{m+1}(r)$, where $A_{i}$ denotes the number of calculated vectors for a given $U_{m+1}(i)$, such that $d\left[U_{m}(i), U_{m}(j)\right] \leq r, j \neq i$.

- Finally, calculate the sample entropy by using $S_{E n}(m, r, N)=-\ln \left[\frac{A^{m+1}(r)}{A^{m}(r)}\right]$ for a tolerance level $r$, where $S_{E n}$ denotes the sample entropy, $m$ is the pattern length and $N$ is the length of the time series.

\section{Complexity Analysis of Time Series}

The MSE approach determines the relative complexity of time series by satisfying the following conditions:

- A time series has a higher complexity than another if it shows higher sample entropy values than those of other time series for the majority of the scale factors.

- A time series is not dynamically complex if its entropy values decrease monotonically with the scale factor. This means that the signal in hand only contains information at the smallest scale. 

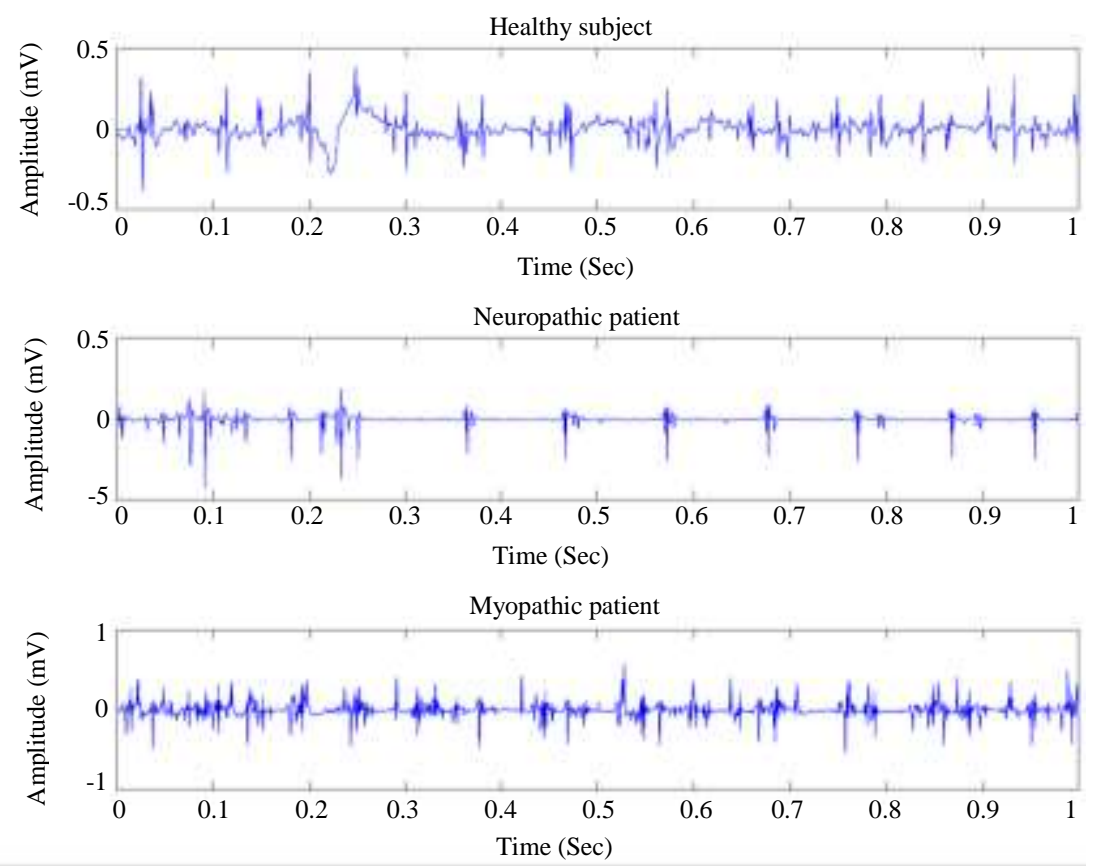

Fig. 1: Time-domain representation of EMG signals for a healthy subject, neuropathic and myopathic patients

\section{Results}

\section{Complexity Analysis of Electromyogram Data}

To calculate the complexity of a time series based on the MSE approach, the values of the three parameters $[\tau$ (time lags), $\mathrm{m}$ (embedding dimensions) and $\mathrm{r}$ (threshold value)] were considered. In this research, the values of the three parameters used to measure SamEn were $m=1, \tau=2$ and $r=0.15 \times$ (standard deviation of the normalized time series) for all three classes (healthy/neuropathic/myopathic).

From Fig. 2, it can be said that higher sample entropy values are observed in the healthy subject rather than patients suffering from neuropathy and myopathy. It is also intuitive that higher complexity is reflected in a healthy subject compared to neuropathic and myopathic patients. It can be inferred that the EMG signals recorded from neuropathic and myopathic patients are more regular and contain less information, thus show less complexity. On the other hand, since the EMG data collected from a healthy patient show less regularity and more information, the healthy subject reveals higher complexity. As the sample entropy values of EMG signals recorded from patients suffering from neuromuscular diseases do not intersect one another in the complexity domain for the majority of the scale factors, these neuromuscular diseases can be detected by the MSE approach in complexity domain. Moreover, healthy subject and patients affected by neuromuscular diseases can also be identified with the MSE technique in the complexity domain due to the nonconvergent behavior of one another.

\section{Statistical Analysis}

In this subsection, the classification results of three groups (healthy, myopathic and neuropathic) acquired in complexity domain are justified based on Support Vector Machine (SVM) and One-way ANOVA test. To do this, 36 mean sample entropy values [12 for each of the class (healthy/neuropathic/myopathic)] are calculated using multiscale entropy approach and used as input to a support vector machine. A 5-fold crossvalidation was used to avoid biased classification performance. The SVM as a classifier validated the classification ability of the MSE approach with statistical parameters (sensitivity, specificity, and accuracy). Applying One-way ANOVA test, statistically significant differences $(p<0.01)$ were observed among the three groups.

According to Table 1, it can be said that one out of twelve mean sample entropy values of healthy records and four out of twelve average sample entropy values of myopathic records were misclassified. On the other hand, eleven out of twelve mean sample entropy values of healthy records, eight out of twelve mean sample entropy values of myopathic records and twelve out of twelve mean sample entropy values of neuropathic records were classified correctly. Table 2 demonstrates that myopathic diseases were identified with a sensitivity of $66.7 \%$ and specificity of $95.8 \%$. Similarly, neuropathic diseases were recognized with a sensitivity of $100 \%$ and specificity of $100 \%$. The healthy, myopathic and neuropathic groups were recognized with a classification accuracy of $86.1 \%$. 


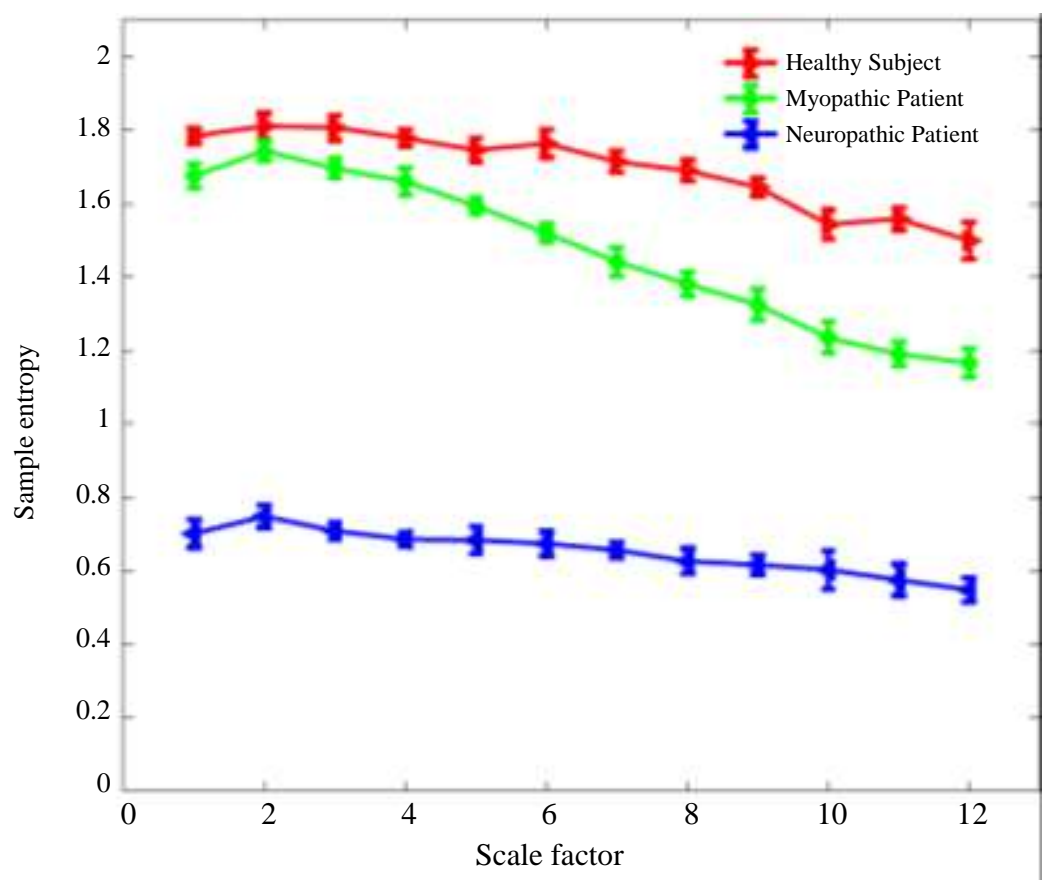

Fig. 2: Multiscale entropy analysis of healthy subject, neuropathic and myopathic patients

Table 1: Confusion Matrix of healthy, neuropathic and myopathic records

\begin{tabular}{llll}
\hline True/predicted & Healthy & Myopathic & Neuropathic \\
\hline Healthy & 11 & 1 & 0 \\
Myopathic & 4 & 8 & 0 \\
Neuropathic & 0 & 0 & 12 \\
\hline
\end{tabular}

Table 2: Sensitivity, specificity and classification accuracy for three groups (healthy/neuropathic/myopathic)

\begin{tabular}{llll}
\hline $\begin{array}{l}\text { Patients } \\
\text { state }\end{array}$ & $\begin{array}{l}\text { Sensitivity } \\
(\%)\end{array}$ & $\begin{array}{l}\text { Specificity } \\
(\%)\end{array}$ & $\begin{array}{l}\text { Classification } \\
\text { accuracy }(\%)\end{array}$ \\
\hline Healthy & 91.7 & 83.3 & 86.1 \\
Myopathic & 66.7 & 95.8 & \\
Neuropathic & 100 & 100 & \\
\hline
\end{tabular}

\section{Discussion}

In this research, the multiscale sample entropy method was applied to the EMG database for identifying neuromuscular diseases. The sample entropy of EMG data was first measured over different scale factors. Multiscale complexity of EMG data was analyzed and characterization of a healthy subject, a neuropathic and a myopathic patient was performed in the complexity domain. Classification results were validated using a support vector machine and one-way ANOVA test.

The complexity of EMG data was measured based on sample entropy. If the sample entropy values of EMG data are higher for the majority of the scale factors, the complexity will be higher. This knowledge was used to explain the classification results in the complexity domain, where the healthy subject exhibited higher complexity compared to neuropathic and myopathic patients. Moreover, a significant difference was reflected in neuropathic and myopathic patients in terms of complexity. These results reveal that EMG signals from the patient with neuropathy exhibit more predictability (more regular, thus less complex) than that of the patient with myopathy. Moreover, a one-way ANOVA test also demonstrated statistically significant differences $(p<0.01)$ among the three classes.

Our experimental results are similar to a previous study (Istenič et al., 2010) that demonstrated that entropy of healthy subjects is always highest and entropy of neuropathic patients is always lowest. The classification accuracy $(86.1 \%)$ of the complexity based MSE method is also promising compared to the amplitude distribution based MSE method (Istenič et al., 2010). Although the complexity-based MSE technique shows promising classification accuracy compared to some studies (Guler and Kocer, 2005; Huppertz et al., 1997), this accuracy is not as promising compared to some other studies (Katsis et al., 2007; Christodoulou and Pattichis, 1999). This is a limitation of our study. Since the database used in this study was collected from only three subjects (one healthy subject, one myopathic and one neuropathic patient), we were only able to analyze 12 subsets of EMG data for each group. In future, we will try to enhance the classification accuracy with the extension of the MSE method. 


\section{Conclusion}

This study characterizes neuromuscular diseases based on multiscale complexity analysis. The multiscale complexity analysis method has shown a promising characterization of neuromuscular diseases in the complexity domain. As a result, this method can be used to classify EMG signals of neuromuscular diseases automatically. Because of its promising classification accuracy, the multiscale entropy method can be used to build a machine based neuromuscular disease detection system.

\section{Author's Contributions}

Kawser Ahammed: Conceived and designed the analysis framework, analyzed the data, drafted the manuscript, read and approved the final manuscript.

Mosabber Uddin Ahmed: Analyzed and interpreted the result, reviewed the manuscript critically, read and approved the final manuscript.

\section{Conflicts of Interest}

All authors affirm the inexistence of conflicts of interest in this study.

\section{References}

Abel, E., P. Zacharia, A. Forster and T. Farrow, 1996. Neural network analysis of the EMG interference pattern. Med. Eng. Phys., 18: 12-17. DOI: 10.1016/1350-4533(95)00032-1

Basmajian, J.V. and C.J. De Luca, 1985. Muscles Alive: Their Functions Revealed by Electromyography. 5th Edn., William and Wilkins, ISBN-10: 068300414X, pp: 561.

Brown, W.F., C.F. Bolton and M.J. Aminoff, 2002. Neuromuscular Function and Disease. 1st Edn., W.B. Saunders, London, ISBN-10: 0721689221, pp: 1948.

Christodoulou, C.I. and C.S. Pattichis, 1999. Unsupervised pattern recognition for the classification of EMG signals. IEEE Trans. Biomed. Eng., 46: 169-178. DOI: 10.1109/10.740879

Costa, A., A.L. Goldberger and C.K. Peng, 2002. Multiscale entropy analysis of complex physiologic time series. Phys. Rev. Lett., 89: 068102-1-068102-4. DOI: 10.1103/PhysRevLett.89.068102

Goldberger, A.L., L.A.N. Amaral, L. Glass, J.M. Hausdorff and P.C. Ivanov et al., 2003. PhysioBank, physiotoolkit and physionet: Components of a new research resource for complex physiologic signals. Circulation, 101: e215-e220. DOI: 10.13026/C24S3D

Guler, N.F. and S. Kocer, 2005. Classification of EMG signals using PCA and FFT. J. Med. Syst., 29: 241-250. DOI: 10.1007/s10916-005-5184-7
Hamilton-Wright, A., D.W. Stashuk and H.R. Tizhoosh, 2007. Fuzzy classification using pattern discovery. IEEE Trans. Fuzzy Syst., 15: 772-783. DOI: $10.1109 /$ TFUZZ.2006.889930

Huppertz, H.J., C. Disselhorst-Klug, J. Silny and G. Rau, 1997. Diagnostic yield of noninvasive high spatial resolution electromyography in neuromuscular diseases. Muscle Nerve, 20: 1360-1370.

DOI:

$10.1002 /($ SICI $) 1097-$ 4598(199711)20:11<1360::AID-MUS3>3.0.CO;2-8

Istenič, R., P.A. Kaplanis and C.S. Pattichis, 2010. Multiscale entropy-based approach to automated surface EMG classification of neuromuscular disorders. Med. Biol. Eng. Comput., 48: 773-781. DOI: $10.1007 / \mathrm{s} 11517-010-0629-7$

Kandel, E.R., J.H. Schwartz and T.M. Jessell, 2000. Principles of Neural Science. 4th Edn., McGrawHill, New York, ISBN-10: 0838577016, pp: 1414.

Katsis, C.D., T.P. Exarchos, C. Papaloukas, Y. Goletsis and D.I. Fotiadis et al., 2007. A two-stage method for MUAP classification based on EMG decomposition. Comput. Biol. Med., 37: 1232-1240. DOI: 10.1016/j.compbiomed.2006.11.010

Katsis, C.D., Y. Goletsis, A. Likas, D.I. Fotiadis and I. Sarmas, 2006. A novel method for automated EMG decomposition and MUAP classification. Artificial Intell. Med., 37: 55-64. DOI: $10.1016 /$ j.artmed.2005.09.002

Kimura, J., 2013. Electrodiagnosis in Diseases of Nerve and Muscle: Principles and Practice. 4th Edn., OUP USA, ISBN-10: 0199738688, pp: 1146.

Kocer, S., 2010. Classification of EMG signals using neuro-fuzzy system and diagnosis of neuromuscular diseases. J. Med. Syst., 34: 321-329.

DOI: $10.1007 / \mathrm{s} 10916-008-9244-7$

Pandey, B. and R.B. Mishra, 2009. An integrated intelligent computing model for the interpretation of EMG based neuromuscular diseases. Expert Syst. Applic., 36: 9201-9213.

DOI: 10.1016/j.eswa.2008.12.023

Richman, J.S. and J.R. Moorman, 2000. Physiological time-series analysis using approximate entropy and sample entropy. Am. J. Physiology-Heart Circulatory Physiol., 278: H2039-2049. DOI: 10.1152/ajpheart.2000.278.6.H2039

Subasi, A., 2013. Classification of EMG signals using PSO optimized SVM for diagnosis of neuromuscular disorders. Comput. Biol. Med., 43: 576-586. DOI: 10.1016/j.compbiomed.2013.01.020

Vallejo, M., C.J. Gallego, L. Duque-Munoz and E. Delgado-Trejos, 2018. Neuromuscular disease detection by neural networks and fuzzy entropy on time-frequency analysis of electromyography signals. Expert Syst., 35: 1-10. DOI: 10.1111/exsy.12274 